\title{
Differential Subordination for Certian Subclasses of $p$-Valent Functions Assoicated with Generalized Linear Operator
}

\author{
R. M. El-Ashwah, ${ }^{1}$ M. K. Aouf, ${ }^{2}$ and S. M. El-Deeb ${ }^{1}$ \\ ${ }^{1}$ Department of Mathematics, Faculty of Science at Damietta, University of Mansoura, New Damietta 34517, Egypt \\ ${ }^{2}$ Department of Mathematics, Faculty of Science, University of Mansoura, Mansoura 33516, Egypt
}

Correspondence should be addressed to S. M. El-Deeb; shezaeldeeb@yahoo.com

Received 14 October 2012; Accepted 20 January 2013

Academic Editor: Liwei Zhang

Copyright (C) 2013 R. M. El-Ashwah et al. This is an open access article distributed under the Creative Commons Attribution License, which permits unrestricted use, distribution, and reproduction in any medium, provided the original work is properly cited.

By making use of the differential subordination analytic functions, we investigate inclusion relationships among certain classes of analytic and $p$-valent functions defined by generalized linear operator.

\section{Introduction}

Let $\mathscr{A}(p)$ denote the class of functions of the form

$$
f(z)=z^{p}+\sum_{k=1}^{\infty} a_{k+p} z^{k+p} \quad(p \in \mathbb{N}=\{1,2, \ldots\}),
$$

which are analytic and $p$-valent in the open unit $\operatorname{disc} U=\{z \in$ $\mathbb{C}:|z|<1\}$.

For functions $f \in \mathscr{A}(p)$, given by (1) and $g$ given by

$$
g(z)=z^{p}+\sum_{k=1}^{\infty} b_{k+p} z^{k+p} \quad(p \in \mathbb{N})
$$

the Hadamard product (or convolution) of $f$ and $g$ is defined by

$$
\begin{aligned}
(f * g)(z) & =z^{p}+\sum_{k=1}^{\infty} a_{k+p} b_{k+p} z^{k+p} \\
& =(g * f)(z) \quad(z \in U ; p \in \mathbb{N}) .
\end{aligned}
$$

For $f, g \in \mathscr{A}(p)$, we say that the function $f$ is subordinate to $g$ denoted by $f(z)<g(z)$, if there exists a Schwarz function $w$, that is, $w \in \mathscr{A}$ with $w(0)=0$ and $|w(z)|<1, z \in U$, such that $f(z)=g(w(z))$ for all $z \in U$. It is well known that, if the function $g$ is univalent in $U$, then $f(z) \prec g(z)$ is equivalent to $f(0)=g(0)$ and $f(U) \subset g(U)$ (see $[1,2])$.
Now, we introduce the linear multiplier operator $\mathscr{L}_{c, p, \lambda}^{m, \delta}:$ $\mathscr{A}(p) \rightarrow \mathscr{A}(p)$ by

$$
\begin{aligned}
& \mathscr{L}_{c, p, \lambda}^{m, \delta} f(z) \\
&=z^{p}+\sum_{k=1}^{\infty}\left(1+\frac{\lambda k}{p}\right)^{m}\left(\frac{c+p}{c+k+p}\right)^{\delta} a_{k+p} z^{k+p} \\
& \quad\left(\delta \geq 0 ; \quad c>-p ; \lambda \geq 0 ; m \in \mathbb{N}_{0}\right) .
\end{aligned}
$$

It is easily verified from (4) that

$$
\begin{aligned}
& z\left(\mathscr{L}_{c, p, \lambda}^{m, \delta} f(z)\right)^{\prime}=(c+p) \mathscr{L}_{c, p, \lambda}^{m, \delta-1} f(z)-c \mathscr{L}_{c, p, \lambda}^{m, \delta} f(z) \\
& \lambda z\left(\mathscr{L}_{c, p, \lambda}^{m, \delta} f(z)\right)^{\prime}=p \mathscr{L}_{c, p, \lambda}^{m+1, \delta} f(z)-p(1-\lambda) \mathscr{L}_{c, p, \lambda}^{m, \delta} f(z) .
\end{aligned}
$$

By specializing the parameters $c, \lambda, \delta$, $p$, and $m$, we obtain the following operators:
(i) $\mathscr{L}_{c, p, 0}^{1, \delta} f(z)=K_{c, p}^{\delta} f(z)$ (see [3]);
(ii) $\mathscr{L}_{a-1,1, \lambda}^{0, \delta} f(z)=L_{a}^{\delta} f(z)($ see $[4,5])$;
(iii) $\mathscr{L}_{1, p, 0}^{1, \delta} f(z)=\mathscr{I}_{p}^{\delta} f(z)($ see $[6,7])$;
(iv) $\mathscr{L}_{c, p, \lambda}^{m, 0} f(z)=D_{\lambda, p}^{m} f(z)$ (see [8]); 
(v) $\mathscr{L}_{c, 1,0}^{1, \delta} f(z)=\mathscr{P}_{c}^{\delta} f(z)($ see $[9,10])$;

(vi) $\mathscr{L}_{c, 1,0}^{1,1} f(z)=\mathscr{L}_{c} f(z)($ see $[11])$;

(vii) $\mathscr{L}_{1,1,0}^{1, \delta} f(z)=\mathscr{J}^{\delta} f(z)$ (see [12]);

(viii) $\mathscr{L}_{c, 1, \lambda}^{m, 0} f(z)=\mathscr{D}_{\lambda}^{m} f(z)($ see $[13])$;

(ix) $\mathscr{L}_{c, 1,1}^{m, 0} f(z)=\mathscr{D}^{m} f(z)($ see $[14])$.

By using the multiplier operator $\mathscr{L}_{c, p, \lambda}^{m, \delta} f(z)$, we define the following classes of functions.

Definition 1. For fixed parameters $A$ and $B$, with $-1 \leq B<$ $A \leq 1$ and $p>\alpha$, we say that the function $f \in \mathscr{A}(p)$ is in the class $\mathcal{S}_{c, p, \lambda}^{m, \delta}(\alpha ; A, B)$ if it satisfies the following subordination condition:

$$
\frac{1}{p-\alpha}\left(\frac{z\left(\mathscr{L}_{c, p, \lambda}^{m, \delta} f(z)\right)^{\prime}}{\mathscr{L}_{c, p, \lambda}^{m, \delta} f(z)}-\alpha\right) \prec \frac{1+A z}{1+B z} .
$$

We note that

(i) $\mathcal{S}_{1, p, 0}^{1,0}(\alpha ; A, B)=\mathcal{S}_{p}^{*}(\alpha ; A, B)$ was studied by Cho et al. [15];

(ii) $\mathcal{S}_{1, p, 0}^{1, \delta}(\alpha ; A, B)=\mathcal{S}_{p}^{\delta}(\alpha ; A, B)$ was studied by Aouf et al. [16];

(iii) $\mathcal{S}_{c, 1, \lambda}^{m, 0}(0 ; A, B)=\mathcal{S}_{\lambda}^{m}(A, B)$ was studied by Patel [17].

For complex numbers $a, b$, and $c$, the Gaussian hypergeometric function is defined by

$$
\begin{gathered}
{ }_{2} F_{1}(a, b, d ; z)=1+\frac{a \cdot b}{d} \frac{z}{1 !}+\frac{a(a+1) \cdot b(b+1)}{d(d+1)} \frac{z^{2}}{2 !}+\cdots \\
=\sum_{k=0}^{\infty} \frac{(a)_{k}(b)_{k}}{(d)_{k}} \frac{z^{k}}{k !}, \quad a, b \in \mathbb{C}, \\
d \in \mathbb{C} \backslash\{0,-1,-2, \ldots\},
\end{gathered}
$$

where $(\lambda)_{k}=\lambda(\lambda+1) \cdots(\lambda+k-1)$ and $(\lambda)_{0}=1$. The series $(8)$ converges absolutely for $z \in U$, hence it represents an analytic function in $U$ (see [18, Chapter 14]).

If $B \neq-1$, from the fact that $h(\bar{z})=\overline{h(z)}, z \in U$, we deduce that the image $h(U)$ is symmetric with respect to the real axis, and that $h$ maps the unit $\operatorname{disc} U$ onto the $\operatorname{disc} \mid w-$ $(1-A B) /\left(1-B^{2}\right) \mid<(A-B) /\left(1-B^{2}\right)$. If $B=-1$, the function $h$ maps the unit disc $U$ onto the half plan $\operatorname{Re} w>(1-A) / 2$, hence we obtain the following.
Remark 2. The function $f \in \mathscr{A}(p)$ is in the class $\mathcal{S}_{c, p, \lambda}^{m, \delta}(\alpha$; $A, B)$ if and only if

$$
\begin{array}{r}
\left|\frac{1}{p-\alpha}\left(\frac{z\left(\mathscr{L}_{c, p, \lambda}^{m, \delta} f(z)\right)^{\prime}}{\mathscr{L}_{c, p, \lambda}^{m, \delta} f(z)}-\alpha\right)-\frac{1-A B}{1-B^{2}}\right|<\frac{A-B}{1-B^{2}}, \\
z \in U, \text { for } B \neq-1,
\end{array}
$$

$$
\begin{array}{r}
\operatorname{Re} \frac{1}{p-\alpha}\left(\frac{z\left(\mathscr{L}_{c, p, \lambda}^{m, \delta} f(z)\right)^{\prime}}{\mathscr{L}_{c, p, \lambda}^{m, \delta} f(z)}-\alpha\right)>\frac{1-A}{2}, \\
z \in U, \text { for } B=-1 .
\end{array}
$$

Definition 3. The function $f \in \mathscr{A}(p)$ is in the class $\mathcal{S}_{c, p, \lambda}^{m, \delta}(\alpha$; $\rho$ ) if it satisfies the following the inequality:

$$
\operatorname{Re} \frac{1}{p-\alpha}\left(\frac{z\left(\mathscr{L}_{c, p, \lambda}^{m, \delta} f(z)\right)^{\prime}}{\mathscr{L}_{c, p, \lambda}^{m, \delta} f(z)}-\alpha\right)>\rho, \quad z \in U,
$$

where $\rho<1$; from (9) and (10) it follows, respectively, that

$$
\begin{gathered}
\mathcal{S}_{c, p, \lambda}^{m, \delta}(\alpha ; A, B) \subset \mathcal{S}_{c, p, \lambda}^{m, \delta}\left(\alpha ; \frac{1-A}{1-B}\right), \\
\mathcal{S}_{c, p, \lambda}^{m, \delta}(\alpha ; A, B)=\mathcal{S}_{c, p, \lambda}^{m, \delta}\left(\alpha ; \frac{1-A}{1-B}\right) \Longleftrightarrow B=-1 .
\end{gathered}
$$

We note that when $c=1$ and $m=0$, the class $\delta_{1, p, 0}^{1, \delta}(\alpha ; \rho)=$ $\mathcal{S}_{p}^{\delta}(\alpha ; \rho)$ was studied by Aouf et al. [16].

Let us consider the first-order differential subordination

$$
H\left(\varphi(z), z \varphi^{\prime}(z)\right) \prec h(z) .
$$

A univalent function $q$ is called its dominant, if $\varphi(z) \prec$ $q(z)$ for all analytic functions $\varphi$ that satisfy this differential subordination. A dominant $\tilde{q}$ is called the best dominant, if $\tilde{q}(z) \prec q(z)$ for all dominants $q$. For the general theory of the first-order differential subordination and its applications, we refer the reader to $[1,2]$.

The object of the present paper is to obtain several inclusion relationships and other interesting properties of functions belonging to the subclasses $\mathcal{S}_{c, p, \lambda}^{m, \delta}(\alpha ; A, B)$ and $\mathcal{S}_{c, p, \lambda}^{m, \delta}(\alpha ; \rho)$ by using the theory of differential subordination.

To establish our main results, we will require the following lemmas.

Lemma 4 (see [19]). Let $\beta, \gamma \in \mathbb{C}$, and let $h$ be a convex function with

$$
\operatorname{Re}[\beta h(z)+\gamma]>0, \quad z \in U
$$

If $p$ is analytic in $U$, with $p(0)=h(0)$, then

$$
p(z)+\frac{z p^{\prime}(z)}{\beta p(z)+\gamma} \prec h(z) \Longrightarrow p(z) \prec h(z) .
$$


Lemma 5 (see [20]). Let $\beta>0, \beta+\gamma>0$, and consider the integral operator $\mathrm{J}_{\beta, \gamma}$ defined by

$$
J_{\beta, \gamma}(f)(z)=\left[\frac{\beta+\gamma}{z^{\gamma}} \int_{0}^{z} f^{\beta}(t) t^{\gamma-1} d t\right]^{1 / \beta},
$$

where the powers are the principal ones.

If $\sigma \in[-\gamma / \beta, 1)$ then the order of starlikeness of the class $J_{\beta, \gamma}\left(S^{*}(\sigma)\right)$, that is, the largest number $\delta(\sigma ; \beta, \gamma)$ such that $J_{\beta, \gamma}\left(S^{*}(\sigma)\right) \subset S^{*}(\delta)$, is given by the number $\delta(\sigma ; \beta, \gamma)=$ $\inf \{\operatorname{Re} q(z): z \in U\}$, where

$$
\begin{gathered}
q(z)=\frac{1}{\beta Q(z)}-\frac{\gamma}{\beta}, \\
Q(z)=\int_{0}^{1}\left(\frac{1-z}{1-t z}\right)^{2 \beta(1-\sigma)} t^{\beta+\gamma-1} d t .
\end{gathered}
$$

Moreover, if $\sigma \in\left[\sigma_{0}, 1\right)$, where $\sigma_{0}=\max \{(\beta-\gamma-$ $1) / 2 \beta ;-\gamma / \beta\}$ and $g=J_{\beta, \gamma}(f)(z)$ with $f \in S^{*}(\sigma)$, then

$$
\operatorname{Re} \frac{z g^{\prime}(z)}{g(z)}>\delta(\sigma ; \beta, \gamma) \quad(z \in U),
$$

where

$$
\delta(\sigma ; \beta, \gamma)=\frac{1}{\beta}\left[\frac{\beta+\gamma}{{ }_{2} F_{1}(1,2 \beta(1-\sigma), \beta+\gamma+1 ; 1 / 2)}-\gamma\right] .
$$

Lemma 6 (see [21]). Let $\phi$ be analytic in $U$ with $\phi(0)=1$ and $\phi(z) \neq 0$ for $0<|z|<1$, and let $A, B \in \mathbb{C}$ with $A \neq B,|B| \leq 1$.

(i) Let $B \neq 0$ and $\gamma \in \mathbb{C}^{*}=\mathbb{C} \backslash\{0\}$ satisfy either $\mid(\gamma(A-$ $B) / B)-1 \mid \leq 1$ or $|(\gamma(A-B) / B)+1| \leq 1$. If $\phi$ satisfies

$$
1+\frac{z \phi^{\prime}(z)}{\gamma \phi(z)} \prec \frac{1+A z}{1+B z}
$$

then

$$
\phi(z) \prec(1+B z)^{\gamma(A-B) / B},
$$

and this is the best dominant.

(ii) Let $\gamma \in \mathbb{C}^{*}$ be such that $|\gamma A|<\pi$, and if $\phi$ satisfies

$$
1+\frac{z \phi^{\prime}(z)}{\gamma \phi(z)} \prec 1+A z
$$

then

$$
\phi(z)<e^{\gamma A z},
$$

and this is the best dominant.

\section{Inclusion Relationships}

Unless otherwise mentioned, we assume throughout this paper that

$$
\begin{aligned}
& -1 \leq B<A \leq 1, \quad p>\alpha, \quad \delta \geq 0, \\
& c>-p, \quad \lambda \geq 0, \quad p \in \mathbb{N}, \quad m \in \mathbb{Z}
\end{aligned}
$$

and the power is the principal one.
Theorem 7. Let

$$
(p-\alpha)(1-A)+(\alpha+c)(1-B) \geq 0 .
$$

(1) Supposing that $\mathscr{L}_{c, p, \lambda}^{m, \delta} f(z) \neq 0$ for all $z \in \dot{U}=U \backslash\{0\}$, then $\mathcal{S}_{c, p, \lambda}^{m, \delta-1}(\alpha ; A, B) \subset \mathcal{S}_{c, p, \lambda}^{m, \delta}(\alpha ; A, B)$.

(2) Moreover, if we suppose in addition that

$$
A \leq 1+\left(\frac{1-B}{p-\alpha}\right)\left(\alpha+\frac{c-p}{2}+\frac{1}{2}\right),
$$

then

$$
\mathcal{S}_{c, p, \lambda}^{m, \delta-1}(\alpha ; A, B) \subset \mathcal{S}_{c, p, \lambda}^{m, \delta}(\alpha ; \rho(p, \alpha, c, A, B)),
$$

where the bound

$$
\begin{aligned}
\rho(p, \alpha, c, A, B) \\
=\frac{1}{p-\alpha} \\
\quad \times\left[\frac{p+c}{{ }_{2} F_{1}(1,2(p-\alpha)(A-B) /(1-B), p+c+1 ; 1 / 2)}\right. \\
\quad \quad-(\alpha+c)],
\end{aligned}
$$

is the best possible.

Proof. Let $f \in \mathcal{S}_{c, p, \lambda}^{m, \delta-1}(\alpha ; A, B)$, and put

$$
g(z)=z\left(\frac{\mathscr{L}_{c, p, \lambda}^{m, \delta} f(z)}{z^{p}}\right)^{1 /(p-\alpha)} \quad(z \in U),
$$

the function $g$ is analytic in $U$, with $g(0)=0$ and $g^{\prime}(0)=1$. Differentiating (29) logarithmically with respect to $z$, we have

$$
\phi(z)=\frac{z g^{\prime}(z)}{g(z)}=\frac{1}{p-\alpha}\left(\frac{z\left(\mathscr{L}_{c, p, \lambda}^{m, \delta} f(z)\right)^{\prime}}{\mathscr{L}_{c, p, \lambda}^{m, \delta} f(z)}-\alpha\right)
$$

$(z \in U)$,

then, using (5) in (30), we obtain

$$
\begin{aligned}
(c+p) & \frac{\mathscr{L}_{c, p, \lambda}^{m, \delta-1} f(z)}{\mathscr{L}_{c, p, \lambda}^{m, \delta} f(z)} \\
= & (p-\alpha) \phi(z)+(\alpha+c) .
\end{aligned}
$$

By differentiating both sides of (31) logarithmically with respect to $z$ and multiplying by $z$, we have

$$
\frac{1}{p-\alpha}\left(\frac{z\left(\mathscr{L}_{c, p, \lambda}^{m, \delta-1} f(z)\right)^{\prime}}{\mathscr{L}_{c, p, \lambda}^{m, \delta-1} f(z)}-\alpha\right)
$$

$$
=\phi(z)+\frac{z \phi^{\prime}(z)}{(p-\alpha) \phi(z)+(\alpha+c)} .
$$


Combining (32) together with $f \in \mathcal{S}_{c, p, \lambda}^{m, \delta-1}(\alpha ; A, B)$, we obtain that the function $\phi$ satisfies the Briot-Bouquet differential subordination as follows:

$$
\begin{aligned}
\phi(z) & +\frac{z \phi^{\prime}(z)}{(p-\alpha) \phi(z)+(\alpha+c)} \\
& \prec \frac{1+A z}{1+B z} \equiv h(z) .
\end{aligned}
$$

Now we will use Lemma 4 for the special case $\beta=p-\alpha$ and $\gamma=\alpha+c$. Since $h$ is a convex function in $U$, a simple computation shows that

$$
\operatorname{Re}\{(p-\alpha) h(z)+(\alpha+c)\}>0, \quad z \in U,
$$

whenever (25) holds, we have $\phi(z) \prec h(z)$; that is, $f \in$ $\mathcal{S}_{c, p, \lambda}^{m, \delta}(\alpha ; A, B)$. If in addition, we suppose that the inequality (26) holds, then all the assumptions of Lemma 5 are verified for the above values of $\beta, \gamma$, and $\sigma=(1-A) /$ $(1-B)$. Then it follows the inclusion $\delta_{c, p, \lambda}^{m, \delta}(\alpha ; A, B) \quad \mathrm{C}$ $\mathcal{S}_{c, p, \lambda}^{m, \delta}(\alpha ; \rho(p, \alpha, c, A, B))$, where the bound $\rho(p, \alpha, c, A, B)$ given by (28) is the best possible.

From Theorem 7, according to the definitions (7) and (11), we deduce the next inclusions.

Corollary 8. Let $p>\alpha$, such that (25) holds.

(1) Supposing that $\mathscr{L}_{c, p, \lambda}^{m, \delta} f(z) \neq 0$ for all $z \in \dot{U}$, then

$$
\mathcal{S}_{c, p, \lambda}^{m, \delta-1}(\alpha ; A, B) \subset \mathcal{S}_{c, p, \lambda}^{m, \delta}(\alpha ; A, B) \subset \mathcal{S}_{c, p, \lambda}^{m, \delta}\left(\alpha ; \frac{1-A}{1-B}\right) .
$$

(2) If we suppose in addition that (26) holds, then

$$
\begin{aligned}
\mathcal{S}_{c, p, \lambda}^{m, \delta-1}(\alpha ; A, B) & \subset \mathcal{S}_{c, p, \lambda}^{m, \delta}(\alpha ; A, B) \\
& \subset \mathcal{S}_{c, p, \lambda}^{m, \delta}(\alpha ; \rho(p, \alpha, c, A, B)),
\end{aligned}
$$

where $\rho(p, \alpha, c, A, B)$ is given by (28). As a consequence of the last inclusion, one has $\rho(p, \alpha, c, A, B) \geq(1-A) /(1-B)$.

For the special case $B=-1$, Theorem 7 reduces to the following.

Corollary 9. Let $a \geq-(c+\alpha) /(p-\alpha)$.

(1) Supposing that $\mathscr{L}_{c, p, \lambda}^{m, \delta} f(z) \neq 0$ for all $z \in \dot{U}$, then

$$
\delta_{c, p, \lambda}^{m, \delta-1}(\alpha ; a) \subset \delta_{c, p, \lambda}^{m, \delta}(\alpha ; a) .
$$

(2) If we suppose in addition that

$$
a \geq \max \left\{-\frac{2 \alpha+c-p+1}{2(p-\alpha)} ;-\frac{\alpha+c}{(p-\alpha)}\right\},
$$

then

$$
\mathcal{S}_{c, p, \lambda}^{m, \delta-1}(\alpha ; a) \subset \mathcal{S}_{c, p, \lambda}^{m, \delta}(\alpha ; \rho(p, \alpha, c, a))
$$

where the bound

$$
\begin{aligned}
\rho(p, \alpha, c, a) & \\
= & \frac{1}{p-\alpha} \\
\quad \times & {\left[\frac{p+c}{{ }_{2} F_{1}(1,2(p-\alpha)(1-a), p+c+1 ; 1 / 2)}\right.} \\
& \quad-(\alpha+c)],
\end{aligned}
$$

is the best possible.

Theorem 10. Let $f \in \mathcal{S}_{c, p, \lambda}^{m, \delta}(\alpha ; \rho)$, where $\rho<1$ and $\rho>\alpha$, then $f \in \mathcal{S}_{c, p, \lambda}^{m, \delta-1}(\alpha ; \rho)$ for $|z|<R(p, \alpha, c, \rho)$, where

$$
\begin{aligned}
& R(p, \alpha, c, \rho)=\min \{r>0: \theta(p, \alpha, c, \rho, r)=0\}, \\
& \theta(p, \alpha, c, \rho, r) \\
& =2 r((1-r)(p-\alpha) \\
& \quad \times \mid(1-\rho)(1-r) \\
& \quad-|\rho+(\alpha+c) /(p-\alpha)|(1+r) \mid)^{-1} .
\end{aligned}
$$

Proof. Since $f \in \mathcal{S}_{c, p, \lambda}^{m, \delta}(\alpha ; \rho)$, the function $u$ given by

$$
u(z)=\frac{1}{p-\alpha}\left(\frac{z\left(\mathscr{L}_{c, p, \lambda}^{m, \delta} f(z)\right)^{\prime}}{\mathscr{L}_{c, p, \lambda}^{m, \delta} f(z)}-\alpha\right) \quad(z \in U),
$$

is analytic in $U$ with $u(0)=1$ and $\operatorname{Re} u(z)>\rho$. Using (5) in (42) and taking the logarithmic differentiation in the resulting equation, we obtain

$$
\begin{aligned}
& \frac{1}{p-\alpha}\left(\frac{z\left(\mathscr{L}_{c, p, \lambda}^{m, \delta-1} f(z)\right)^{\prime}}{\mathscr{L}_{c, p, \lambda}^{m, \delta-1} f(z)}-\alpha\right) \\
& \quad=u(z)+\frac{z u^{\prime}(z)}{(p-\alpha) u(z)+(\alpha+c)} .
\end{aligned}
$$

If we denote $k(z)=(u(z)-\rho) /(1-\rho)$, then $k(0)=1$ and Re $k(z)>0, z \in U$ and substituting in (43) we obtain

$$
\begin{aligned}
\frac{1}{p-\alpha} & \left(\frac{z\left(\mathscr{L}_{c, p, \lambda}^{m, \delta-1} f(z)\right)^{\prime}}{\mathscr{L}_{c, p, \lambda}^{m, \delta-1} f(z)}-\alpha\right)-\rho \\
= & (1-\rho) \\
& \times[k(z)
\end{aligned}
$$

$$
\left.+\frac{z k^{\prime}(z)}{(p-\alpha)(1-\rho) k(z)+\rho(p-\alpha)+(\alpha+c)}\right],
$$


hence

$$
\begin{aligned}
& \operatorname{Re} \frac{1}{p-\alpha}\left(\frac{z\left(\mathscr{L}_{c, p, \lambda}^{m, \delta-1} f(z)\right)^{\prime}}{\mathscr{L}_{c, p, \lambda}^{m, \delta-1} f(z)}-\alpha\right)-\rho \\
& \geq(1-\rho) \\
& \quad \times {\left[\operatorname{Re} k(z)-\left(\left|z k^{\prime}(z)\right|\right)((p-\alpha)\right.} \\
&\left.\quad \times|(1-\rho) k(z)-| \rho+((\alpha+c) /(p-\alpha))||)^{-1}\right] .
\end{aligned}
$$

By using the well-known results [22]

$$
\begin{aligned}
\left|z k^{\prime}(z)\right| \leq \frac{2 r}{1-r^{2}} \operatorname{Re} k(z), \quad \operatorname{Re} k(z) & \geq \frac{1-r}{1+r}, \\
|z| & =r<1,
\end{aligned}
$$

together with the inequality (45), we get

$$
\begin{aligned}
\operatorname{Re} & \frac{1}{p-\alpha}\left(\frac{z\left(\mathscr{L}_{c, p, \lambda}^{m, \delta-1} f(z)\right)^{\prime}}{\mathscr{L}_{c, p, \lambda}^{m, \delta-1} f(z)}-\alpha\right)-\rho \\
& \geq(1-\rho)[1-\theta(p, \alpha, c, \rho, r)] \operatorname{Re} k(z),|z|=r .
\end{aligned}
$$

Since the right hand side term of the inequality (47) is nonnegative whenever $|z| \leq R(p, \alpha, c, \rho)$ is given by (41), using the fact that the real part of an analytic function is harmonic, we deduce that $f \in \mathcal{S}_{c, p, \lambda}^{m, \delta-1}(\alpha ; \rho)$ for $|z|<R(p, \alpha, c, \rho)$.

For a function $f \in \mathscr{A}(p)$, let the integral operator $F_{\mu, p}$ : $\mathscr{A}(p) \rightarrow \mathscr{A}(p)$ defined by Saitoh [23] and Saitoh et al. [24]

$$
\begin{aligned}
F_{\mu, p}(f(z))= & \frac{\mu+p}{z^{\mu}} \int_{0}^{z} t^{\mu-1} f(t) d t \\
= & z^{p}+\sum_{k=p+1}^{\infty} \frac{\mu+p}{k+\mu} a_{k} z^{k} \\
= & \left(z^{p}+\sum_{k=p+1}^{\infty} \frac{\mu+p}{k+\mu} z^{k}\right) * f(z) \\
= & z^{p}{ }_{2} F_{1}(1, \mu+p ; \mu+p+1 ; z) \\
& * f(z) \quad(z \in U ; \mu>-p) .
\end{aligned}
$$

From (4) and (48), we have

$$
\begin{gathered}
z\left(\mathscr{L}_{c, p, \lambda}^{m, \delta} F_{\mu, p}(f(z))\right)^{\prime} \\
\quad=(\mu+p) \mathscr{L}_{c, p, \lambda}^{m, \delta} f(z)-\mu \mathscr{L}_{c, p, \lambda}^{m, \delta} F_{\mu, p}(f(z)), \\
\mathscr{L}_{c, p, \lambda}^{m, \delta} F_{\mu, p}(f(z))=F_{\mu, p}\left(\mathscr{L}_{c, p, \lambda}^{m, \delta} f(z)\right), \quad f \in A(p) .
\end{gathered}
$$

We now prove the next result.
Theorem 11. Let $\alpha+\mu>0$ and

$$
(\alpha+\mu)(1-B)+(p-\alpha)(1-A) \geq 0
$$

(i) Supposing that $F_{\mu, p}(f(z)) \neq 0$ for all $z \in \dot{U}=U \backslash\{0\}$, then

$$
F_{\mu, p}\left(\mathcal{S}_{c, p, \lambda}^{m, \delta}(\alpha ; A, B)\right) \subset \mathcal{S}_{c, p, \lambda}^{m, \delta}(\alpha ; A, B) .
$$

(ii) Moreover, if we suppose in addition that

$$
A \leq 1+\left(\frac{1-B}{p-\alpha}\right) \min \left\{\frac{\mu+2 \alpha-p+1}{2} ; \mu+\alpha\right\},
$$

then

$$
F_{\mu, p}\left(\mathcal{S}_{c, p, \lambda}^{m, \delta}(\alpha ; A, B)\right) \subset \mathcal{S}_{c, p, \lambda}^{m, \delta}(\alpha ; r(p, \alpha, \mu, A, B)),
$$

where the bound

$r(p, \alpha, \mu, A, B)$

$$
\begin{aligned}
& =\frac{1}{p-\alpha} \\
& \times\left[\frac{p+\mu}{{ }_{2} F_{1}(1,2(p-\alpha)(A-B) /(1-B), \mu+p+1 ; 1 / 2)}\right. \\
& \quad-(\mu+\alpha)],
\end{aligned}
$$

is the best possible.

Proof. Let $f \in \mathcal{S}_{c, p, \lambda}^{m, \delta}(\alpha ; A, B)$, and suppose that $F_{\mu, p}(f(z)) \neq 0$ for all $z \in \dot{U}$. Let

$$
g(z)=z\left(\frac{\mathscr{L}_{c, p, \lambda}^{m, \delta} F_{\mu, p}(f(z))}{z^{p}}\right)^{1 /(p-\alpha)}
$$

$$
(z \in U)
$$

then $g$ is analytic in $U$, with $g(0)=0$ and $g^{\prime}(0)=1$. Taking the logarithmic differentiation in (55), we have

$$
\phi(z)=\frac{z g^{\prime}(z)}{g(z)}=\frac{1}{p-\alpha}\left(\frac{z\left(\mathscr{L}_{c, p, \lambda}^{m, \delta} F_{\mu, p}(f(z))\right)^{\prime}}{\mathscr{L}_{c, p, \lambda}^{m, \delta} F_{\mu, p}(f(z))}-\alpha\right)
$$

Now, by using (49) in (56), we obtain

$$
(p+\mu) \frac{\mathscr{L}_{c, p, \lambda}^{m, \delta} f(z)}{\mathscr{L}_{c, p, \lambda}^{m, \delta} F_{\mu, p}(f(z))}=(p-\alpha) \phi(z)+(\mu+\alpha) .
$$


By differentiating in both sides of (57) logarithmical with respect to $z$ and multiplying by $z$, we have

$$
\begin{aligned}
& \frac{1}{p-\alpha}\left(\frac{z\left(\mathscr{L}_{c, p, \lambda}^{m, \delta} f(z)\right)^{\prime}}{\mathscr{L}_{c, p, \lambda}^{m, \delta} f(z)}-\alpha\right) \\
& =\phi(z)+\frac{z \phi^{\prime}(z)}{(p-\alpha) \phi(z)+(\mu+\alpha)} .
\end{aligned}
$$

Since $f \in \mathcal{S}_{c, p, \lambda}^{m, \delta}(\alpha ; A, B)$, from (58), we obtain that the function $\phi$ satisfies the Briot-Bouquet differential subordination

$$
\phi(z)+\frac{z \phi^{\prime}(z)}{(p-\alpha) \phi(z)+(\mu+\alpha)}<\frac{1+A z}{1+B z} \equiv h(z) .
$$

Now we will use Lemma 4 for the special case $\beta=p-\alpha$ and $\gamma=\mu+\alpha$; we have $\phi(z) \prec h(z)$, that is, $F_{\mu, p} \in$ $\delta_{c, p, \lambda}^{m, \delta}(\alpha ; A, B)$. If we suppose in addition that the inequality (52) holds, then all the assumptions of the Lemma 5 are satisfied for $\beta, \gamma$, and $\sigma=(1-A) /(1-B)$, hence it follows the inclusion $F_{\mu, p}\left(\mathcal{S}_{c, p, \lambda}^{m, \delta}(\alpha ; A, B)\right) \subset \mathcal{S}_{c, p, \lambda}^{m, \delta}(\alpha ; r(p, \alpha, \mu, A, B))$, and the bound $r(p, \alpha, \mu, A, B)$ given by (54) is the best possible.

Taking $B=-1$ in Theorem 11, we obtain the next corollary.

Corollary 12. Let $\alpha+\mu>0$ and $a \geq-(\mu+\alpha) /(p-\alpha)$.

(1) Supposing that $\mathscr{L}_{c, p, \lambda}^{m, \delta} F_{\mu, p}(f(z)) \neq 0$ for all $z \in \dot{U}$, then

$$
F_{\mu, p}\left(\delta_{c, p, \lambda}^{m, \delta}(\alpha ; a)\right) \subset \delta_{c, p, \lambda}^{m, \delta}(\alpha ; a) .
$$

(2) If we suppose in addition that

$$
a \geq \max \left\{-\frac{\mu+2 \alpha-p+1}{2(p-\alpha)} ;-\frac{\mu+\alpha}{p-\alpha}\right\},
$$

then

$$
F_{\mu, p}\left(\mathcal{S}_{c, p, \lambda}^{m, \delta}(\alpha ; a)\right) \subset \mathcal{S}_{c, p, \lambda}^{m, \delta}(\alpha ; r(p, \alpha, \mu, a)),
$$

where the bound

$$
\begin{aligned}
r(p, \alpha, \mu, a) & \\
= & \frac{1}{p-\alpha} \\
& \times\left[\frac{\mu+p}{{ }_{2} F_{1}(1,2(p-\alpha)(1-a), \mu+p+1 ; 1 / 2)}\right. \\
& \quad-(\mu+\alpha)],
\end{aligned}
$$

is the best possible.
Theorem 13. Let $v \in \mathbb{C}^{*}$, and let $A, B \in \mathbb{C}$ with $A \neq B$ and $|B| \leq 1$. Suppose that

$$
\begin{gathered}
\left|\frac{v(c+p)(A-B)}{B}-1\right| \leq 1 \quad \text { or } \\
\left|\frac{v(c+p)(A-B)}{B}+1\right| \leq 1, \quad \text { if } B \neq 0, \\
|v A| \leq \frac{\pi}{c+p}, \quad \text { if } B=0 .
\end{gathered}
$$

If $f \in \mathscr{A}(p)$ with $\mathscr{L}_{c, p, \lambda}^{m, \delta} f(z) \neq 0$ for all $z \in \dot{U}$, then

$$
\frac{\mathscr{L}_{c, p, \lambda}^{m, \delta-1} f(z)}{\mathscr{L}_{c, p, \lambda}^{m, \delta} f(z)} \prec \frac{1+A z}{1+B z}
$$

implies

$$
\left(\frac{\mathscr{L}_{c, p, \lambda}^{m, \delta} f(z)}{z^{p}}\right)^{v} \prec q_{1}(z),
$$

where

$$
q_{1}(z)= \begin{cases}(1+B z)^{\nu(c+p)(A-B) / B} & \text { if } B \neq 0, \\ e^{\nu(c+p) A z} & \text { if } B=0,\end{cases}
$$

is the best dominant.

Proof. Let us put

$$
\phi(z)=\left(\frac{\mathscr{L}_{c, p, \lambda}^{m, \delta} f(z)}{z^{p}}\right)^{v} \quad(z \in U),
$$

then $\phi$ is analytic in $U$, with $\phi(0)=1$ and $\phi(z) \neq 0$ for all $z \in \dot{U}$. By differentiating both sides of (68) logarithmical with respect to $z$ and using (5), we have

$$
1+\frac{z \phi^{\prime}(z)}{v(c+p) \phi(z)}=\frac{\mathscr{L}_{c, p, \lambda}^{m, \delta-1} f(z)}{\mathscr{L}_{c, p, \lambda}^{m, \delta} f(z)}
$$

$$
\prec \frac{1+A z}{1+B z} \text {. }
$$

Now the assertions of Theorem 13 follows by using Lemma 6 for the special case $\gamma=v(c+p)$.

Putting $B=-1$ and $A=1-2 \rho, 0 \leq \rho<1$, in Theorem 13, we obtain the following corollary.

Corollary 14. Assume that $v \in \mathbb{C}^{*}$ satisfies either

$$
\begin{gathered}
|2 v(c+p)(1-\rho)-1| \leq 1 \quad \text { or } \\
|2 v(c+p)(1-\rho)+1| \leq 1, \quad \text { if } B \neq 0,
\end{gathered}
$$

If $f \in \mathscr{A}(p)$ with $\mathscr{L}_{c, p, \lambda}^{m, \delta} f(z) \neq 0$ for all $z \in \dot{U}$, then

$$
\operatorname{Re} \frac{\mathscr{L}_{c, p, \lambda}^{m, \delta-1} f(z)}{\mathscr{L}_{c, p, \lambda}^{m, \delta} f(z)}>\rho
$$


implies

$$
\left(\frac{\mathscr{L}_{c, p, \lambda}^{m, \delta} f(z)}{z^{p}}\right)^{v} \prec q_{2}(z)=(1-z)^{-2 v(c+p)(1-\rho)},
$$

and $q_{2}$ is the best dominant.

\section{Properties Involving}

\section{the Multiplier Operator $\mathscr{L}_{c, p, \lambda}^{m, \delta}$}

Theorem 15. If $f \in \mathcal{S}_{c, p, \lambda}^{m, \delta}(\alpha ; A, B)$, then for all $s, t \in \mathbb{C}$ with $|s| \leq 1,|t| \leq 1$ and $s \neq t$, the next subordination holds

$$
\frac{t^{p} \mathscr{L}_{c, p, \lambda}^{m, \delta} f(z s)}{s^{p} \mathscr{L}_{c, p, \lambda}^{m, \delta} f(z t)} \prec \begin{cases}\left(\frac{1+B z s}{1+B z t}\right)^{(p-\alpha)(A-B) / B}, & \text { for } B \neq 0 \\ \exp [(p-\alpha) A z(s-t)], & \text { for } B=0 .\end{cases}
$$

Proof. If $f \in \mathcal{S}_{c, p, \lambda}^{m, \delta}(\alpha ; A, B)$, from (7) it follows that

$$
\frac{z\left(\mathscr{L}_{c, p, \lambda}^{m, \delta} f(z)\right)^{\prime}}{\mathscr{L}_{c, p, \lambda}^{m, \delta} f(z)} \prec \frac{p+[p B+(p-\alpha)(A-B)] z}{1+B z} \equiv k(z) .
$$

Moreover, the function $k$ defined by (74) and the function $h$ given by

$$
h(z) \equiv h(z ; s, t)=\int_{0}^{z}\left(\frac{s}{1-s u}-\frac{t}{1-t u}\right) d u
$$

are convex in $U$. By combining a general subordination theorem [25, Theorem 4] with (74), we get

$$
\begin{gathered}
\left(\frac{z\left(\mathscr{L}_{c, p, \lambda}^{m, \delta} f(z)\right)^{\prime}}{\mathscr{L}_{c, p, \lambda}^{m, \delta} f(z)}-p\right) * h(z) \\
\prec \frac{(p-\alpha)(A-B) z}{1+B z} * h(z) .
\end{gathered}
$$

For every analytic function $\phi$ in $U$ with $\phi(0)=0$, we have

$$
\phi(z) * h(z)=\int_{t z}^{s z} \frac{\phi(u)}{u} d u,
$$

and thus, from (76) and (77), we deduce

$$
\begin{aligned}
\int_{t z}^{s z}( & \left(\frac{\left.\mathscr{L}_{c, p, \lambda}^{m, \delta} f(u)\right)^{\prime}}{\mathscr{L}_{c, p, \lambda}^{m, \delta} f(u)}-\frac{p}{u}\right) d u \\
& <(p-\alpha)(A-B) \int_{t z}^{s z} \frac{d u}{1+B u} .
\end{aligned}
$$

This last subordination implies

$$
\begin{array}{r}
\exp \left(\int_{t z}^{s z}\left(\frac{\left(\mathscr{L}_{c, p, \lambda}^{m, \delta} f(u)\right)^{\prime}}{\mathscr{L}_{c, p, \lambda}^{m, \delta} f(u)}-\frac{p}{u}\right) d u\right) \\
<\exp \left((p-\alpha)(A-B) \int_{t z}^{s z} \frac{d u}{1+B u}\right),
\end{array}
$$

and by simplification, we get the assertion of Theorem 15 .
Corollary 16. If $f \in \mathcal{S}_{c, p, \lambda}^{m, \delta}(\alpha ; A, B)$, then for $|z|=r<1$, the next inequalities hold

$$
\begin{gathered}
\left|\mathscr{L}_{c, p, \lambda}^{m, \delta} f(z)\right| \leq \begin{cases}r^{p}(1+B r)^{(p-\alpha)(A-B) / B}, & \text { for } B \neq 0, \\
r^{p} \exp [(p-\alpha) A r], & \text { for } B=0,\end{cases} \\
\left|\mathscr{L}_{c, p, \lambda}^{m, \delta} f(z)\right| \geq \begin{cases}r^{p}(1-B r)^{(p-\alpha)(A-B) / B}, & \text { for } B \neq 0, \\
r^{p} \exp [-(p-\alpha) A r], & \text { for } B=0,\end{cases} \\
\left|\arg \frac{\mathscr{L}_{c, p, \lambda}^{m, \delta} f(z)}{z^{p}}\right| \\
\leq \begin{cases}\frac{(p-\alpha)(A-B)}{|B|} \sin ^{-1}(|B| r), & \text { for } B \neq 0, \\
(p-\alpha) A r, & \text { for } B=0 .\end{cases}
\end{gathered}
$$

All of the estimates asserted here are sharp.

Proof. Taking $s=1$ and $t=0$ in (73) and using the definition of subordination, we obtain

$$
\frac{\mathscr{L}_{c, p, \lambda}^{m, \delta} f(z)}{z^{p}}= \begin{cases}(1+B w(z))^{(p-\alpha)(A-B) / B}, & \text { for } B \neq 0 \\ \exp [(p-\alpha) A w(z)], & \text { for } B=0\end{cases}
$$

where $w$ is analytic function in $U$ with $w(0)=0$ and $|w(z)| \leq$ 1 for $z \in U$. According to the well-known Schwarz's theorem, we have $|w(z)| \leq|z|$ for all $z \in U$.

(i) If $B>0$, then we find from (83) that

$$
\begin{aligned}
\left|\frac{\mathscr{L}_{c, p, \lambda}^{m, \delta} f(z)}{z^{p}}\right| & =\exp \left[\frac{(p-\alpha)(A-B)}{B} \log |1+B w(z)|\right] \\
& =|1+B w(z)|^{(p-\alpha)(A-B) / B} \\
& \leq(1+B r)^{(p-\alpha)(A-B) / B} .
\end{aligned}
$$

(ii) If $B<0$, we can easily obtain

$$
\begin{aligned}
\left|\frac{\mathscr{L}_{c, p, \lambda}^{m, \delta} f(z)}{z^{p}}\right| & =|1+B w(z)|^{(p-\alpha)(A-B) /-B} \\
& \leq\left[(1+B r)^{-1}\right]^{(p-\alpha)(A-B) /-B} \\
& =(1+B r)^{(p-\alpha)(A-B) / B} .
\end{aligned}
$$

This proves the inequality (80) for $B \neq 0$. Similarly, we can prove the other inequalities in (80) and (81). Now, for $|z|=r$ and $B \neq 0$, we observe from (83) that

$$
\begin{aligned}
\left|\arg \frac{\mathscr{L}_{c, p, \lambda}^{m, \delta} f(z)}{z^{p}}\right| & =\frac{(p-\alpha)(A-B)}{|B|}|\arg (1+B w(z))| \\
& \leq \frac{(p-\alpha)(A-B)}{|B|} \sin ^{-1}(|B| r),
\end{aligned}
$$


It is easy to see that all of the estimates in Corollary 16 are sharp, being attained by the function $f_{0}$ defined by

$$
\mathscr{L}_{c, p, \lambda}^{m, \delta} f_{0}(z)= \begin{cases}z^{p}(1+B z)^{(p-\alpha)(A-B) / B}, & \text { for } B \neq 0, \\ z^{p} \exp [(p-\alpha) A z], & \text { for } B=0 .\end{cases}
$$

Remark 17. (i) Putting $c=1$ and $m=0$ in our results, we obtain the results obtained by Aouf et al. [16].

(ii) By specializing the parameters $c, \lambda, \delta, p$, and $m$, we obtain various results for different operators defined in Section 1 .

\section{Acknowledgment}

The authors thank the referees for their valuable suggestions which led to improvement of this paper.

\section{References}

[1] T. Bulboaca, Differential Subordinations and Superordinations, Recent Results, Hous of Scientific Book, Cluj-Napoca, Romania, 2005.

[2] S. S. Miller and P. T. Mocanu, Differential Subordinations: Theory and Applications, Series on Monographs and Texbooks in Pure and Applied Mathematics, no. 225, Marcel Dekker, New York, NY, USA, 2000.

[3] S. M. Khairnar and M. More, "On a subclass of multivalent $\beta$-uniformly starlike and convex functions defined by a linear operator," IAENG International Journal of Applied Mathematics, vol. 39, no. 3, pp. 1-9, 2009.

[4] M. K. Aouf, "Some inclusion relationships associated with the Komatu integral operator," Mathematical and Computer Modelling, vol. 50, no. 9-10, pp. 1360-1366, 2009.

[5] M. K. Aouf, "The Komatu integral operator and strongly closeto-convex functions," Bulletin of Mathematical Analysis and Applications, no. 3, pp. 209-219, 2011.

[6] S. Shams, S. R. Kulkarni, and J. M. Jahangiri, "Subordination properties for $p$-valent functions defined by integral operator," International Journal of Mathematics and Mathematical Sciences, vol. 2006, Article ID 94572, 3 pages, 2006.

[7] A. Ebadian, S. Shams, Z. G. Wang, and Y. Sun, "A class of multivalent analytic functions involving the generalized Jung-KimSrivastava operator," Acta Universitatis Apulensis, vol. 18, pp. 265-277, 2009.

[8] M. K. Aouf, R. M. El-Ashwah, and S. M. El-Deeb, "Some inequalities for certain $p$-valent functions involving extended multiplier transformations," Proceedings of the Pakistan Academy of Sciences, vol. 46, no. 4, pp. 217-221, 2009.

[9] Y. Komatu, "On analytic prolongation of a family of integral operators," Mathematica (Cluj), vol. 32, no. 55, pp. 141-145, 1990.

[10] R. K. Raina and I. B. Bapna, "On the starlikeness and convexity of a certain integral operator," Southeast Asian Bulletin of Mathematics, vol. 33, pp. 101-108, 2009.

[11] S. D. Bernardi, "Convex and starlike univalent functions," Transactions of the American Mathematical Society, vol. 135, pp. 429-446, 1969.

[12] A. Ebadian and S. Najafzadeh, "Uniformly starlike and convex univalent functions by using certain integral operator," Acta Universitatis Apulensis, vol. 20, pp. 17-23, 2009.
[13] F. M. Al-Oboudi, "On univalent functions defined by a generalized Salagean operator," International Journal of Mathematics and Mathematical Sciences, vol. 27, pp. 1429-1436, 2004.

[14] G. Sălăgean, "Subclasses of univalent functions," in Complex Analysis. 5th Romanian-Finnish Seminar, vol. 1013 of Lecture Notes in Mathematics, pp. 362-372, Springer, Berlin, Germany, 1983.

[15] N. E. Cho, O. S. Kwon, and H. M. Srivastava, "Inclusion relationships and argument properties for certain subclasses of multivalent functions associated with a family of linear operators," Journal of Mathematical Analysis and Applications, vol. 292, no. 2, pp. 470-483, 2004.

[16] M. K. Aouf, T. Bulboaca, and A. O. Mostafa, "Subordination properties of subclasses of $p$-valent functions involving certain operators," Publicationes Mathematicae, vol. 73, no. 3-4, pp. 401416, 2008.

[17] J. Patel, "Inclusion relations and convolution properties of certain sub-classes of analytic functions defined by a generalized Salagean operator," Bulletin of the Belgian Mathematical Society Simon Stevin, vol. 15, pp. 33-47, 2008.

[18] E. T. Whittaker and G. N. Watson, A Course of Modern Analysis: An Introduction to the General Theory of infinite Processes and of Analytic Functions; With an Account of the Principal Transcendental Functions, Cambridge University Press, Cambridge, UK, 4th edition, 1927.

[19] P. J. Eenigenburg, S. S. Miller, P. T. Mocanu, and M. O. Reade, "On a Briot-Bouquet differential subordination," in General Inequalities 3, vol. 64 of International Series of Numerical Mathematics, pp. 339-348, Birkhauser, Basel, Switzerland, 1983.

[20] P. T. Mocanu, D. Ripeanu, and I. Serb, “The order of starlikeness of certain integral operators," Mathematica (Cluj), vol. 23(46), no. 2, pp. 225-230, 1981.

[21] M. Obradovic and S. Owa, "On certain properties for some classes of starlike functions," Journal of Mathematical Analysis and Applications, vol. 145, no. 2, pp. 357-364, 1990.

[22] T. H. Macgregor, "Radius of univalence of certain analytic functions," Proceedings of the American Mathematical Society, vol. 14, pp. 514-520, 1963.

[23] H. Saitoh, "On certain class of multivalent functions," Mathematica Japonica, vol. 37, pp. 871-875, 1992.

[24] H. Saitoh, S. Owa, T. Sekine, M. Nunokawa, and R. Yamakawa, "An application of a certain integral operator," Applied Mathematics Letters, vol. 5, no. 2, pp. 21-24, 1992.

[25] St. Ruscheweyh and T. Sheil-Small, "Hadamard products of Schlicht functions and the Pólya-Schoenberg conjecture," Commentarii Mathematici Helvetici, vol. 48, no. 1, pp. 119-135, 1973. 


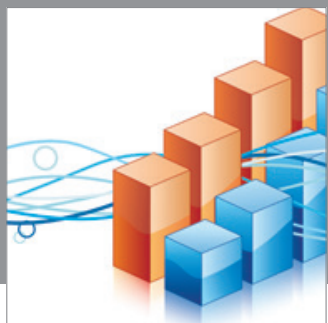

Advances in

Operations Research

mansans

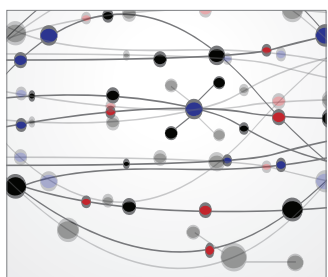

The Scientific World Journal
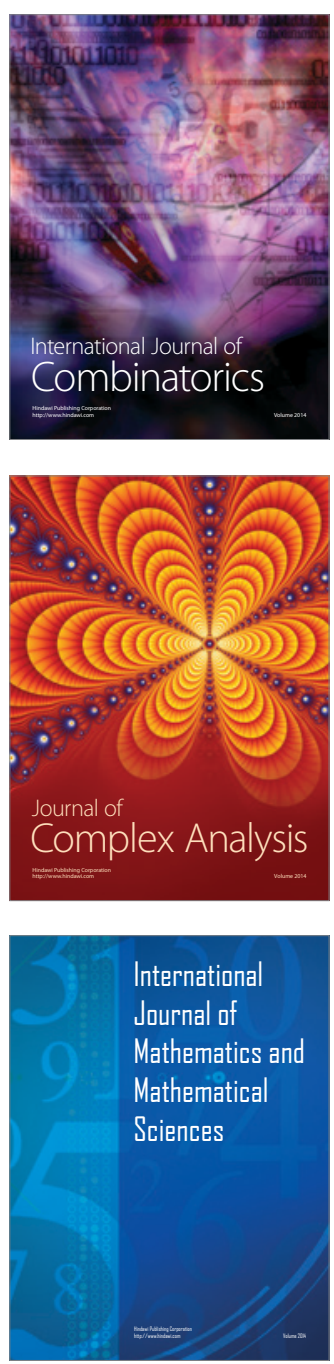
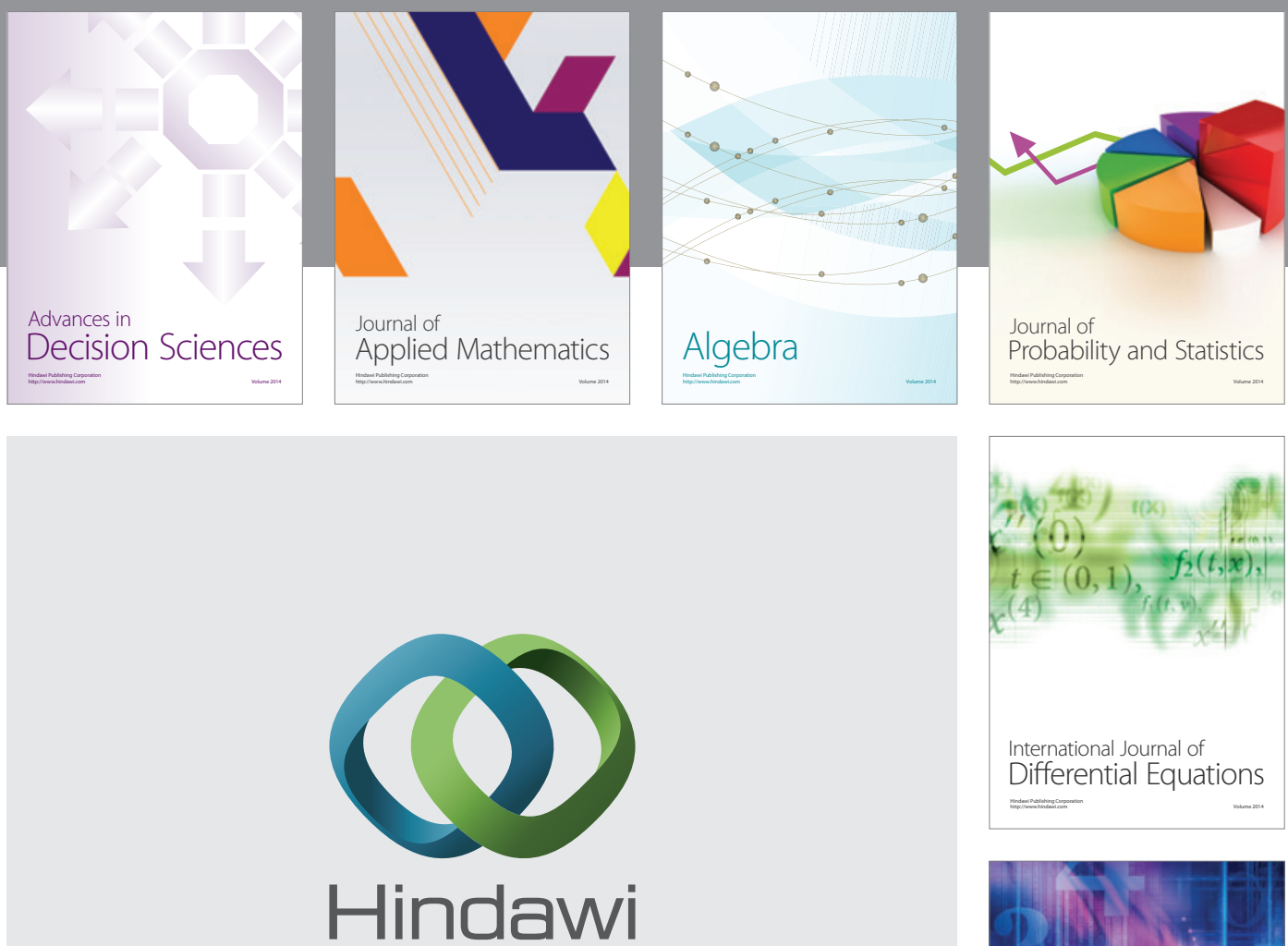

Submit your manuscripts at http://www.hindawi.com
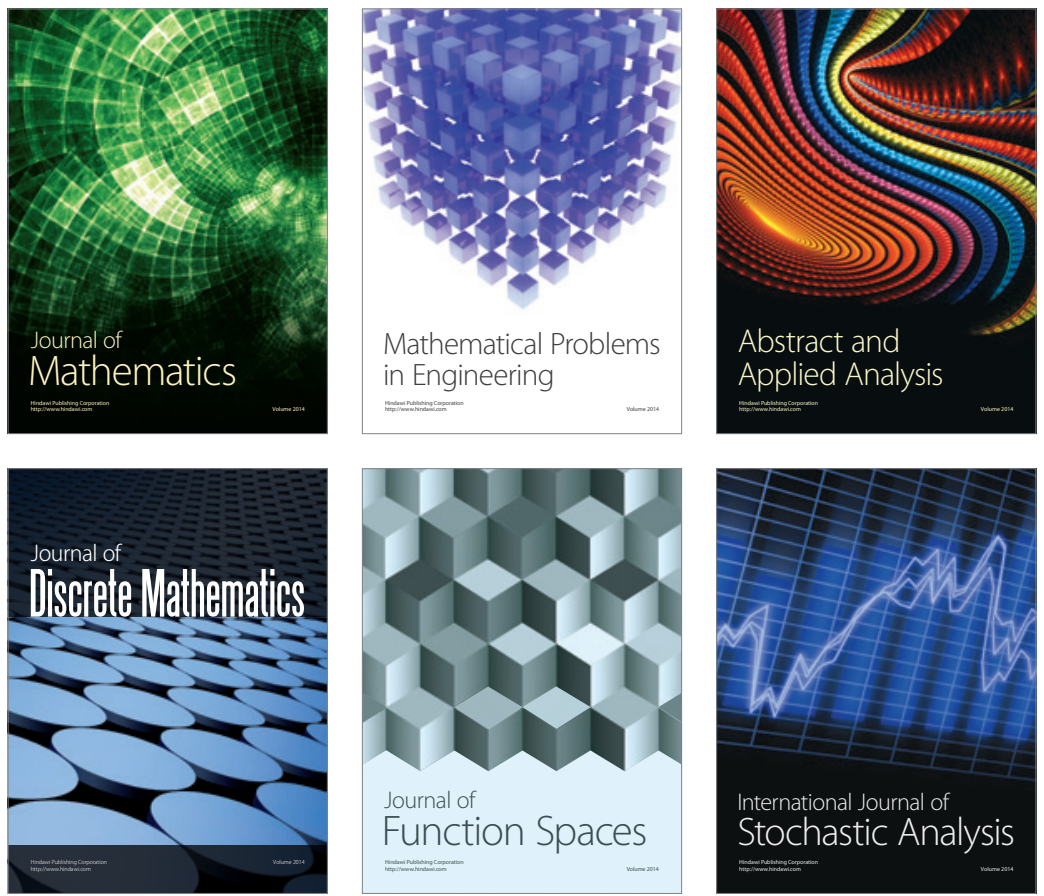

Journal of

Function Spaces

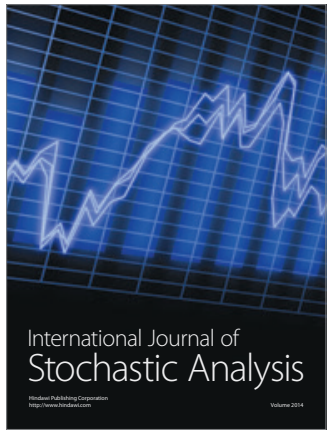

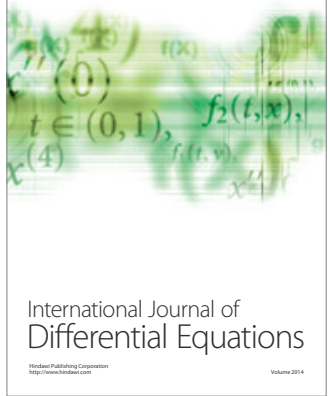
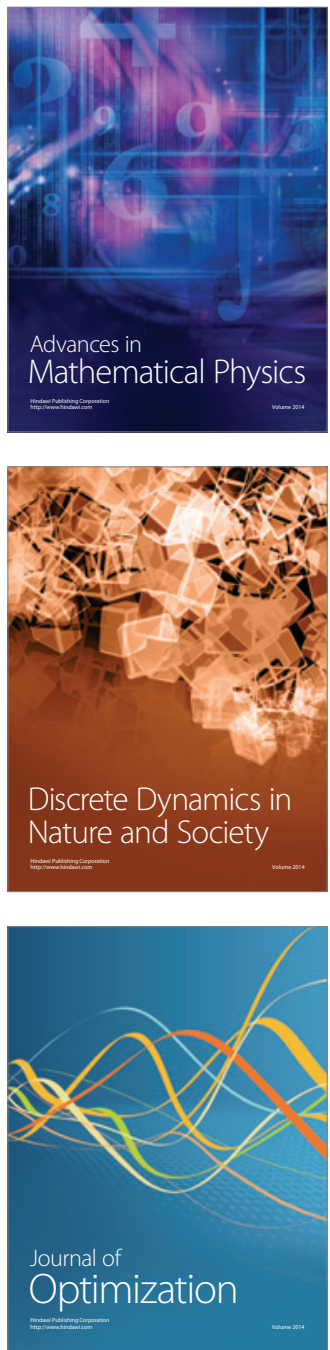